\title{
ENSEÑANZA DE LA QUÍMICA DESDE LAS CTS PARA EL DESARROLLO DE ACTITUDES FAVORABLES EN LOS ESTUDIANTES
}

\author{
Leidy Bibiana Ariza Traslaviña ${ }^{1}$ \\ dqu910_lariza@pedagogica.edu.co \\ Cesar Augusto Amaya Posse ${ }^{1}$ \\ dqu909_camaya@pedagogica.edu.co
}

\begin{abstract}
Resumen
Dentro del proceso de enseñanza es frecuente encontrar que uno de las principales dificultades es la relacionada con las actitudes desfavorables de los estudiantes en el campo del conocimiento de la química; esta dificultad tiene varios factores que lo producen, así como posibles soluciones, de esta manera se diseña y aplica una estrategia que ofrece alternativas para enseñar conceptos de la química en educación secundaria, bajo un enfoque CTS (Ciencia, Tecnología y Sociedad), cuyo objeto permite establecer relaciones, entre el conocimiento adquirido por medio del trabajo y problemáticas del entorno, así como el desarrollo de actitudes positivas hacia la química. Se aplican lecturas diseñadas a partir de información recopilada, implementación de mini-proyectos y dos instrumentos, uno diseñado para esta investigación y otro ya validado en otro trabajo. Se logra que los estudiantes de grado décimo y once del Instituto Pedagógico Nacional establezcan relaciones entre los conceptos de química y problemáticas ambientales y sociales.
\end{abstract}

\section{Palabras Clave}

Actitudes hacia la química, Relaciones CTS, Aprendizaje.

\footnotetext{
1 Estudiante en formación de Licenciatura en Química, Universidad Pedagógica Nacional
} 


\section{REVISIÓN CONCEPTUAL}

El estudio de las actitudes en los estudiantes respecto al aprendizaje no es algo nuevo, trae una historia que tiene sus inicios en las investigaciones sobre los errores y esquemas conceptuales alternativos. A partir de ellas se buscaba encontrar formas para un cambio conceptual que generara estructuras mentales más complejas, adaptadas a la realidad. Fishbein y Ajzen, presentan, hacia la década de 1960, la Teoría de la Acción Razonada en la cual exponen que la actitud se relaciona directamente con la conducta. Reyes (2007), traduce a cómo relacionar la actitud con una intención, que permitiría predecir una futura conducta, (Ruiz, Saura \& Minguez, 1993).

El término actitud se define de muchas formas, se ha confundido con expresiones como creencia, rasgo y hábito, Fishbein y Ajzen (1960), explican la distinción entre actitud y creencia como:"una referencia a las evaluaciones personales favorables o desfavorables de los objetos; las creencias presentan la información que se tiene acerca de los objetos" (Mora, 1997, p. 20-31).

Aún existe una amplia discusión acerca del concepto actitud, sin embargo Shrigley, Montmollin y Moscovici en Mora (1997), luego de un análisis exhaustivo sobre el concepto "actitud", llegaron a establecer que son características adquiridas (pueden depender del aprendizaje), e implican una relación entre las personas y los objetos, es decir se forman en reacción con algo (persona, grupo, institución); una actitud se corresponde con una acción, las influencias sociales afectan las actitudes y a partir de estas es posible predecir la conducta, esta investigación parte de este enunciado.

Según Furio \& Gil (1989) la importancia del estudio sobre las actitudes hacia la ciencia y su aprendizaje radica en que este es uno los problemas más graves que aqueja la enseñanza de las ciencias naturales por lo que debe ser un tema esencial en la didáctica de las mismas.
Es pertinente referir que las actitudes se pueden clasificar de dos formas: actitudes hacia la ciencia y actitudes científicas. En este trabajo se caracterizan las actitudes hacia las ciencias en las que se encuentran las actitudes hacia su aprendizaje. Investigaciones realizadas por expertos, sobre las mismas, muestran claramente tres componentes: cognitivo, afectivo y conductual; el componente cognitivo representa la comprensión o el conocimiento que tiene o cree tener una persona; el afectivo representa la tendencia del estudiante a mostrar acuerdo o desacuerdo, es el componente que más influencia tiene sobre la actitud y no es identificable a simple vista; y el componente conductual o de acción muestra las acciones y el comportamiento en una situación. Las actitudes frente al aprendizaje de las ciencias muestran la influencia, especialmente del componente afectivo, y del posicionamiento adoptado por los estudiantes respecto a preferencias académicas concretas (las disciplinas científicas, las carreras afines a la ciencia, las actividades de laboratorio, etc.), así como de su creencia y sentimiento. En este sentido, las actitudes como predisposiciones con componentes cognitivos y conductuales, pero sobre todo emotivos, pueden ser positivos y negativos, hacia un determinado objeto de actitud (Manassero \& Vasquez, 2001).

\section{RELACIONES CTS}

El estudio del movimiento Ciencia, Tecnología y Sociedad en América Latina es abordado a partir de las teorías desarrolladas por Vaccarezza (1998).

Los autores en este movimiento plantean que la evolución de CTS en América Latina ha pasado de un status de movimiento al de campo, de tal manera que tiene una constitución multidisciplinar en el abordaje de determinados objetos o problemas sociales. La panorámica de CTS en Latinoamérica se concreta en la variedad de objetivos y problemas de análisis que componen la matriz disciplinar de este campo de trabajo. En esta matriz, se incluyen las políticas científicas y tecnológicas en América Latina, gestión de tecnología, los 
procesos de innovación, el cambio técnico en la empresa, el progreso de las disciplinas y comunidades científicas, los problemas de la vinculación en ciencia-producción, el comercio internacional de la tecnología, la articulación en el análisis de la perspectiva de la ciencia jurídica y de la economía y por último la prospectiva tecnológica.

\section{DESCRIPCIÓN DEL PROBLEMA}

Con frecuencia, los estudiantes no tienen una actitud favorable hacia las ciencias, ni tampoco hacia una comprensión creativa e innovadora de los problemas cotidianos (Polania, 2011). Esto conlleva a que muchas ideas que se pueden originar en el aula de clase se pierdan, y necesariamente se llegue a las mismas conclusiones siempre a partir de la inclusión del mismo contenido.

Las actitudes de los estudiantes en la clase de química del IPN de diferentes cursos $\left(10^{\circ}\right.$ y $\left.11^{\circ}\right)$ frecuentemente son de indiferencia y de apatía, lo cual según Reyes (2007) está necesariamente reflejando su conducta, y por lo tanto, se puede entender como actitudes negativas hacia la ciencia. Esto particularmente se debe a varios factores que implica la clase, haciendo hincapié en que uno de esos factores, es la forma de abordar los conceptos en la clase, ya que son totalmente desarticulados del contexto del estudiante, causando que estos desarrollen actitudes poco favorables.

Por consiguiente se plantea la pregunta de investigación: ¿Cómo por medio del abordaje de cuestiones sociales se fomenta el desarrollo favorable de las actitudes hacia el aprendizaje de la química de los estudiantes del I.P.N.?

\section{OBJETIVO GENERAL}

- Fortalecer y desarrollar actitudes favorables hacia la clase de química en los estudiantes de grado decimo y once del IPN, desde las relaciones CTS (Ciencia, Tecnología y Sociedad).

\section{OBJETIVOS ESPECÍFICOS}

- Evaluar las actitudes de los estudiantes en la clase de química, identificando si positivas o negativas.

- Identificar actitudes favorables de los estudiantes por medio del abordaje de situaciones problemáticas.

- Determinar cómo se favorecen las actitudes positivas en los estudiantes con la implementación de mini-proyectos.

\section{METODOLOGÍA}

La realización de este proyecto se lleva a cabo en tres fases según el siguiente esquema:

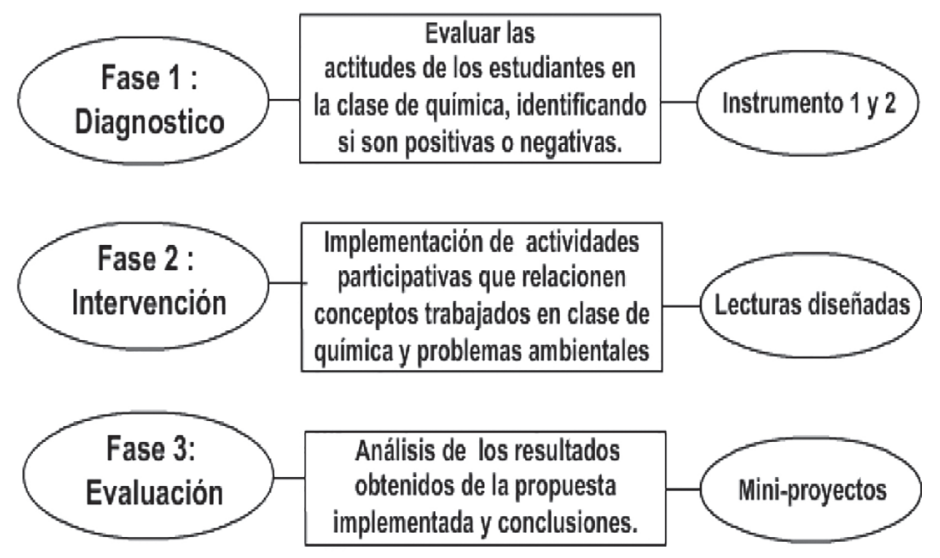


Ilustración 1. Metodología

\section{RESULTADOSY ANÁLISIS}

\section{INSTRUMENTO 1}

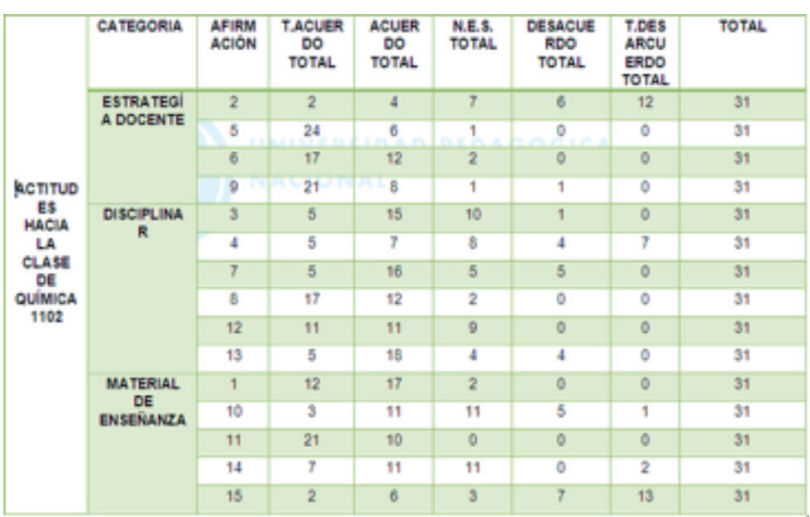

Tabla 1. Resultados instrumento 1, 1102

\begin{tabular}{|c|c|c|c|c|c|c|c|c|}
\hline \multirow{16}{*}{$\begin{array}{c}\text { ACTITLO } \\
\text { ES } \\
\text { HACUA } \\
\text { LA } \\
\text { CLASE } \\
\text { DE } \\
\text { Quimica } \\
1003\end{array}$} & CATEGCEAA & $\begin{array}{l}\text { AfireaA } \\
\text { Cion }\end{array}$ & $\begin{array}{l}\text { TACUER } \\
\text { DO } \\
\text { TOTAL }\end{array}$ & $\begin{array}{l}\text { ACUEFO } \\
\text { OTOTAL }\end{array}$ & $\begin{array}{l}\text { NE.S. } \\
\text { TOTAL }\end{array}$ & $\begin{array}{l}\text { DESACUER } \\
\text { DO TOTAL }\end{array}$ & $\begin{array}{l}\text { TLESA } \\
\text { ROUER } \\
\text { DO } \\
\text { TOTAL }\end{array}$ & TOTAL \\
\hline & \multirow{4}{*}{$\begin{array}{l}\text { ESTRATEGI } \\
\text { A DOCENTE }\end{array}$} & 2 & 4 & 8 & 15 & 4 & 6 & 37 \\
\hline & & S & 17 & 15 & 8 & 0 & 0 & 37 \\
\hline & & 6 & 10 & 16 & 7 & 4 & 0 & 37 \\
\hline & & 9 & 17 & 18 & 1 & 1 & 0 & 37 \\
\hline & \multirow{6}{*}{$\underset{\mathrm{R}}{\text { DISCIPLA }}$} & 3 & 4 & 12 & 12 & 7 & 2 & 37 \\
\hline & & 4 & 0 & 1 & 9 & 5 & 22 & 37 \\
\hline & & 7 & 10 & 13 & 7 & 6 & 1 & 37 \\
\hline & & 8 & 12 & 19 & 8 & 1 & 0 & 37 \\
\hline & & 12 & 7 & 18 & 9 & 3 & 0 & 37 \\
\hline & & 13 & 2 & 16 & 12 & 6 & 1 & 37 \\
\hline & \multirow{5}{*}{$\begin{array}{c}\text { MATERIAL } \\
\text { DE } \\
\text { ENSERANZ } \\
\text { A }\end{array}$} & 1 & 10 & 18 & 8 & 1 & 0 & 37 \\
\hline & & 10 & $\$$ & 12 & 18 & 4 & 1 & 37 \\
\hline & & 11 & 22 & 13 & 2 & 0 & 0 & 37 \\
\hline & & 14 & 5 & 11 & 17 & 3 & 1 & 37 \\
\hline & & 15 & 3 & 4 & 8 & 14 & 7 & 36 \\
\hline
\end{tabular}

Tabla 2. Resultados instrumento 1, 1003

A partir de los resultados que se muestran en la tablas 1 y 2, es preciso afirmar que el problema de actitud en los estudiantes es causado por la manera cómo están presentados los contenidos en química, ya que a pesar del esfuerzo de parte del docente por relacionar la temática de química con el entorno conocido por el estudiante, se necesita fortalecer los conocimientos que este adquiere para poder explicar con mayor fluidez lo que ocurre a su alrededor. Está ubicado dentro de los rangos presentados en las tablas de resultados para la aplicación del instrumento 1, tanto para grado once, como para decimo, pues los valores más altos los tiene la categoría disciplinar; lo cual refleja que para los estudiantes es importante conocer a fondo las causas de los fenómenos que ocurren en su entorno, aunque no dejan de lado el material o la estrategia que usa el profesor; esto no es tan relevante ya que no es la causa de las actitudes negativas que toman frente a la clase de química

De esta manera se procede a la aplicación de las actividades propuestas en ambos niveles, cuyo objetivo principal es lograr establecer relaciones entre el conocimiento que adquieren, con cada problema de tipo social, económico, sanitario, ambiental, entre otros, que pueda presentar el manejo de materiales, recursos y productos en su entorno.

\section{INSTRUMENTO 2}

\section{ÍNDICE GLOBAL DE ACTITUD}

\begin{tabular}{|l|l|l|}
\hline & 1003 & 1102 \\
\hline 10111 & 0,07 & 0,30 \\
\hline 10211 & 0,07 & $-0,01$ \\
\hline 10412 & $-0,29$ & $-0,35$ \\
\hline 10413 & 0,20 & 0,27 \\
\hline
\end{tabular}

Tabla 3. Resultados Instrumento 2.

Los resultados obtenidos de todos los estudiantes se llevan a una matriz de registro, en la que se realiza el cambio de las respuestas por unos valores determinados según el tipo de idea adecuada, plausible, o ingenua, y los valores se introducen en la ecuación sugerida en Manassero \& Vasquez (2001) que consiste en obtener el índice de actitud global, el cual se puede encontrar entre - 1 y 1 , y su distribución en estos valores serán interpretados como características positivas o negativas, hacia las concepciones de ciencia, la tecnología y la sociedad.

Si el índice es positivo, la actitud es valiosa, tanto mejor cuanto más se acerca al valor de unidad. Si el índice es negativo, la actitud es ingenua, tanto más ingenua cuanto más se acerca al valor de unidad negativa. 


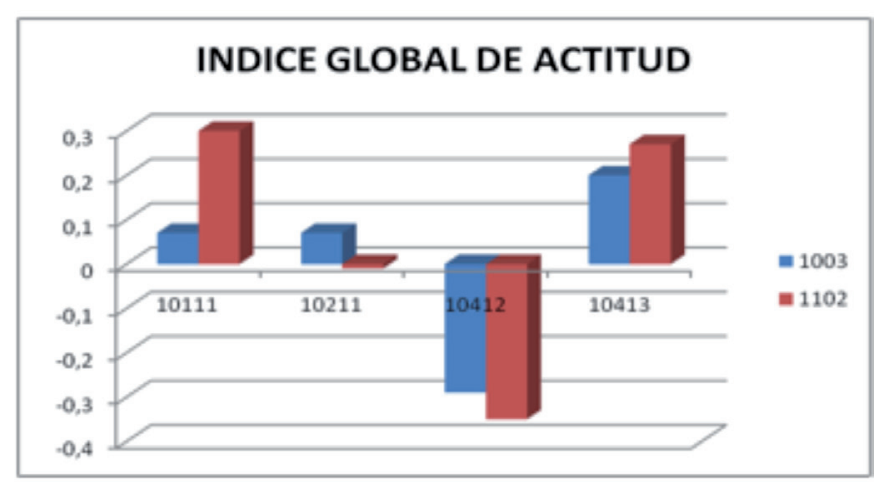

Gráfica 1. Índice global de actitud.

Hallando el índice de actitud global en cada una de las cuatro preguntas realizadas para los grados décimo (1003) y once (1102), los estudiantes presentan un índice de actitud global mayor que cero, lo que indica que respecto a las definiciones de ciencia pueden manifestar una actitud positiva siendo esta mayor para los estudiantes de grado once.

En los aspectos donde se indaga por la definición de tecnología, los índices son muy cercanos a cero, es decir, la actitud es ingenua o poco favorable y no está muy alejada entre los dos cursos. Asimismo donde se relaciona la influencia de la ciencia en la tecnología, los índices son negativos para los dos grupos, probablemente porque creen que la ciencia está al servicio de la tecnología y finalmente, la pregunta donde se relaciona la influencia de la tecnología en la ciencia, los índices son favorables para los dos grupos evidenciándose una mejor tendencia en el grado once.

\section{ACTIVIDADES DÉCIMOY ONCE}

Se establecen conexiones entre los fenómenos que se estudian en clase y los diferentes problemas que generan, tanto el procesamiento del petróleo, como la lluvia ácida en el ambiente; estas conexiones son definidas como ideas que son categorizadas dentro del marco ciencia-sociedad y ciencia-ambiente. Las respuestas que los estudiantes escriben reflejan la importancia de mostrar una realidad muy unida al conocimiento que reciben en el aula de clase, además, en el ejerci- cio de exponer los puntos de vista al respecto, se observa bastante interés en la participación y una acentuada atención por las respuestas de sus compañeros.

Asimismo durante la discusión, los estudiantes están atentos a los comentarios de sus compañeros y de las posibles preguntas que surgen, se logra captar la atención de la mayoría de los estudiantes y tener una mayor participación en la actividad realizada; de esta manera, relacionan la situación expuesta con los problemas sociales, y también se consigue que relacionen los problemas ambientales con las temáticas de reacciones químicas (decimo) e hidrocarburos (once).

\section{MINI-PROYECTOS}

Se realizan en dos momentos: el primer momento, donde los estudiantes deben consultar y proponer la metodología para su desarrollo; el segundo momento, es la entrega del documento final.

Se logra establecer que los estudiantes son conscientes de la acción de la humanidad en los problemas socio-ambientales y que los intereses económicos también inciden de forma importante; en este caso, los estudiantes tratan de reflejar en sus escritos su interés por problemáticas locales. En general, los estudiantes se ven motivados por las consultas y la entrega de los documentos.

En cuanto a las actitudes, se observa que definitivamente, es necesario un enfoque diferente a la manera como se enseña la química, es decir, que las actividades relacionen aspectos de su vida y de la sociedad, para fortalecer el cambio de actitud y por tanto, de comportamiento hacia el aprendizaje de las ciencias. Con propuestas como estas se logra que el estudiante piense y actué de forma crítica frente a su realidad y por lo tanto actué en búsqueda de respuestas y explicaciones a los fenómenos que actualmente los afectan. 


\section{CONCLUSIONES}

- La propuesta implementada logra que los estudiantes muestren una actitud favorable hacia la clase de química y establezcan relaciones entre CTS.

- Con la aplicación de las lecturas relacionadas a problemáticas socio-ambientales como la Iluvia acida, el petróleo y las consecuencias de su explotación, se establecen relaciones entre temas de la química y la realidad del avance científico.
- El desarrollo de mini-proyectos por parte de los estudiantes a partir de posibles problemáticas socio-ambientales planteadas o indagadas por ellos, logra que afiancen su conocimiento acerca de la temáticas trabajadas durante el semestre, como la nomenclatura química orgánica (1102) e inorgánica (1003), además de las generalidades de los hidrocarburos (1102) y reacciones químicas (1003), que llevan a cuestionarse sobre el desarrollo científico y su influencia en la sociedad, tomando posturas críticas y reflexivas.

\section{BIBLIOGRAFÍA}

Escudero, T., \& Lacasta, E. (1984). Las actitudes cientificas de los futuros maestros en relacion con sus conocimientos. Enseñanza de las Ciencias.

Furio, M., \& Gil, D. (1989). La didactica de las ciencias en la formacion inicial del profesorado: una orientacion y un programa teoricamente fundamentados. Enseñanza de las Ciencias.

Manassero, M. A., \& Vasquez, A. (2001). Intrumentos y metodos para la evaluacion de las actitudes relacionadas con la ciencia, la tecnologia y la sociedad. Investigacion Didactica.

Martínez Perez, L. F., Peña, D. C., \& Villamil, Y. M. (2007). Relaciones ciencia, tecnología, sociedad y ambiente a partir de casos simulados: una experiencia en la enseñanza de la Química. Ciëncia \& Ensino.

Mora, W. (1997). Actitudes hacia la imagen de las ciencias. Actualidad educativa.
Polania, Y. C. (2011). Aprendizaje basado en problemas: una perspectiva didactica para la formacion de actitud cientifica en la enseñanza de las ciencias naturales. Colombia.

Reyes, L. (07 de Septiembre de 2007). Alfaguia. Recuperado el 23 de Septiembre de 2012, de http:// www.alfaguia.org/alfaguia/files/1320437914_40. pdf

Ruiz, P., Saura, J. P., \& Minguez, R. (1993). La formacion de actitudes positivas hacia el estudio de las ciencias experimentales. Revista de Educacion.

Vasconcelos, C., Praia, J., \& Almeida, L. (2005). Actitudes y hábitos de estudio en ciencias naturales: validacion de una escala y su utilización práctica. Enseñanza de las ciencias. 


\section{ANEXOS}

\section{INSTRUMENTO 1}

Marque con una X la casilla que usted considere más adecuada de acuerdo a la frase que se le indica en cada caso, según la escala indicada a continuación:

\begin{tabular}{|c|l|}
\hline Totalmente de acuerdo & 5 \\
\hline De acuerdo & 4 \\
\hline No estoy seguro & 3 \\
\hline En desacuerdo & 2 \\
\hline Totalmente en desacuerdo & 1 \\
\hline
\end{tabular}

\begin{tabular}{|l|l|l|l|l|l|}
\hline & 5 & 4 & 3 & 2 & 1 \\
\hline $\begin{array}{l}\text { Las guías y talleres en clase son adecuadas y constituyen un gran } \\
\text { complemento para el aprendizaje autónomo. }\end{array}$ & & & & \\
\hline $\begin{array}{l}\text { Los temas de química se hacen difíciles por la manera como el profesor } \\
\text { aborda la clase. }\end{array}$ & & & & \\
\hline Los temas que se ven en química son fáciles. & & & & & \\
\hline En su mayoría, la química se relaciona con la carrera que seguiré. & & & & & \\
\hline La evaluación de los temas siempre está de acuerdo a lo visto en clase. & & & & & \\
\hline La manera como dirige la clase el profesor es apropiada. & & & & \\
\hline Se requiere aprender varios conceptos de memoria. & & & & & \\
\hline En casa es común encontrar fenómenos químicos y físicos. & & & & \\
\hline Todas las dudas relacionadas con la clase son atendidas. & & & & \\
\hline Se debería abordar más lecturas y realizar discusiones del tema. & & & & \\
\hline La práctica de laboratorio es un espacio útil para experimentar la teoría. & & & & & \\
\hline Siempre se aprende algo nuevo y aplicable en la vida durante la clase. & & & & & \\
\hline Es necesario conocer los procesos químicos implicados en el diario vivir. & & & & & \\
\hline $\begin{array}{l}\text { El espacio de clase de química es un escenario apto para la discusión de } \\
\text { problemáticas actuales. }\end{array}$ & & & & \\
\hline La química es una ciencia que muy poco aporta a la sociedad. & & & & & \\
\hline
\end{tabular}

Responda las siguientes preguntas

1. ¿Al finalizar el colegio piensas continuar tus estudios? ¿Qué carrera estudiaras?

2. ¿Encuentra alguna relación de las temáticas trabajadas en clase de química, con la profesión que piensa desempeñar durante la vida? Explique su respuesta. 


\section{INSTRUMENTO 2}

Este instrumento no es de carácter evaluativo, pero requiere de su sinceridad al momento de responder, solo debe marcar un numero que exprese su grado de acuerdo con el enunciado que se presenta.

10111 Deffinir qué es la ciencia es difficil porgue ésta es algo complejo y engloba muchas cosas. Pero la ciencia principalmente es:

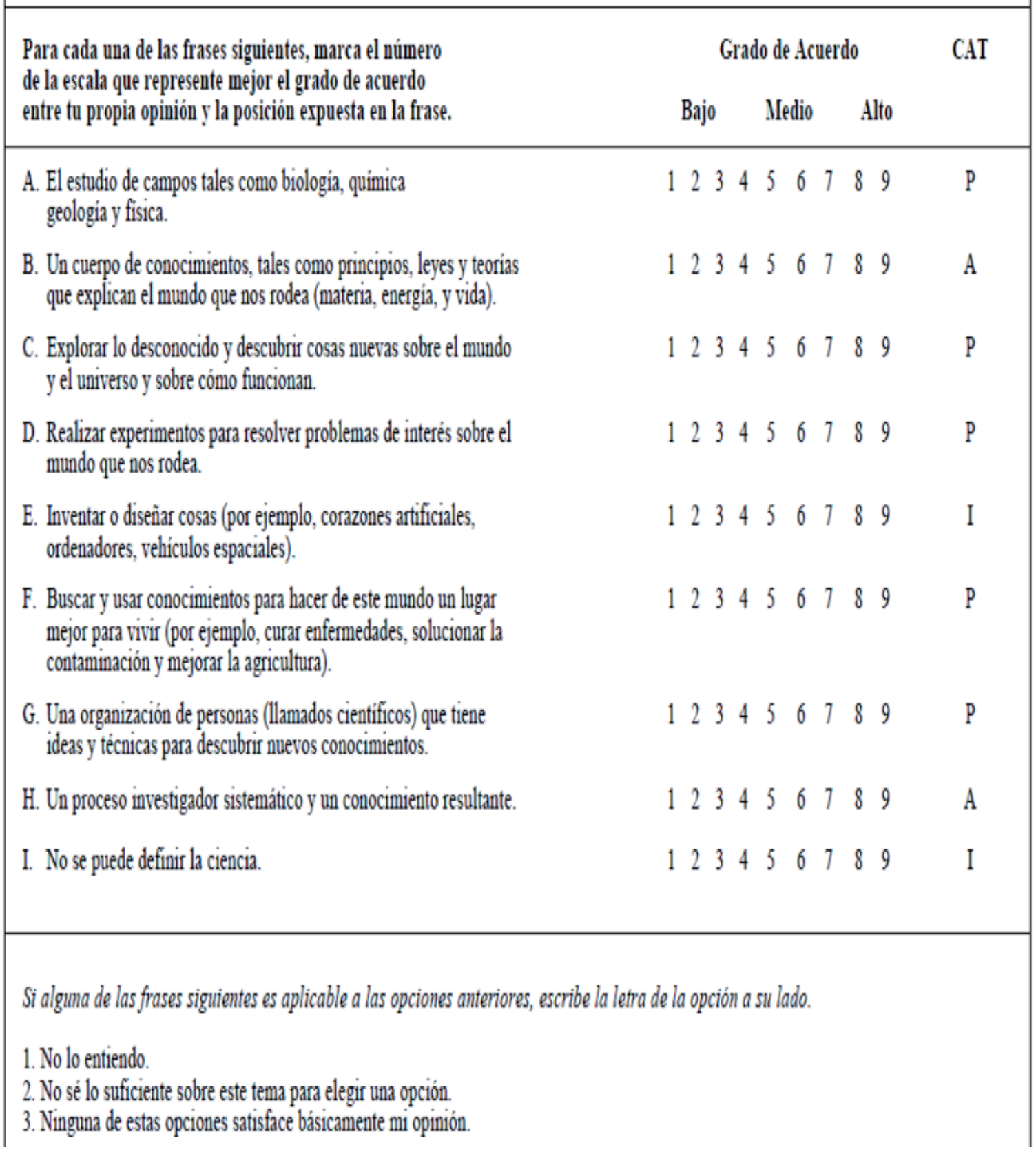




\title{
EJEMPLO LECTURA
}

\author{
Asignatura: Química \\ Recopilado por: A C \\ Licenciatura en Química
}

\section{EL PETRÓLEO EN COLOMBIA}

Una refinería es un enorme complejo donde el petróleo crudo se somete, en primer lugar, a un proceso de destilación o separación física y luego a procesos químicos que permiten extraerle buena parte de la gran variedad de los componentes que contiene. El petróleo tiene una gran variedad de compuestos, hasta el punto de que de él pueden obtenerse más de dos mil productos. El petróleo igualmente puede clasificarse en cuatro categorías: parafínico, nafténico, asfáltico o mixto y aromático.

Los productos que se sacan del proceso de refinación se llaman derivados y los hay de dos tipos: los combustibles, como la gasolina, A.C.P.M., etc.; y los petroquímicos, tales como polietileno, benceno, etc. Las refinerías son muy distintas unas de otras, según las tecnologías y los esquemas de proceso que se utilicen, así como su capacidad. Las hay para procesar crudos livianos, crudos pesados o mezclas de ambos. Por consiguiente, los productos que se obtienen varían de una a otra. La refinación se cumple en varias etapas. Es por esto por lo que una refinería tiene numerosas torres, unidades, equipos y tuberías. Es algo así como una ciudad de plantas de proceso.

En Colombia hay dos refinerías: El Complejo Industrial de Barrancabermeja, propiedad de Ecopetrol, ubicada en el centro del país, en el departamento de Santander y la Refinería de Cartagena, cuyos propietarios son Glencore International y Ecopetrol y está ubicada en la Zona Industrial de Mamonal, a 10 minutos del centro histórico de Cartagena de Indias en la Costa Atlántica.

A la Refinería de Barrancabermeja se le llama "Complejo Industrial" porque además de producir combustibles como la gasolina y el GLP, también produce petroquímicos e industriales. En térmi- nos sencillos, una refinería de este tipo funciona de la siguiente manera:

El primer paso de la refinación del petróleo crudo se cumple en las torres de "destilación primaria" o "destilación atmosférica”. En su interior, estas torres operan a una presión cercana a la atmosférica y están divididas en numerosos compartimientos a los que se denominan "bandejas" o "platos". Cada bandeja tiene una temperatura diferente y cumple la función de fraccionar los componentes del petróleo.

El crudo llega a estas torres después de pasar por un horno, donde se "cocina" a temperaturas de hasta $400{ }^{\circ} \mathrm{C}$ que lo convierten en vapor. Esos vapores entran por la parte inferior de la torre de destilación y ascienden por entre las bandejas. A medida que suben pierden calor y se enfrían. Cuando cada componente vaporizado encuentra su propia temperatura, se condensa y se deposita en su respectiva bandeja, a la cual están conectados ductos por los que se recogen las distintas corrientes que se separaron en esta etapa.

Al fondo de la torre cae el "crudo reducido", es decir, aquel que no alcanzó a evaporarse en esta primera etapa. Se cumple así el primer paso de la refinación. De abajo hacia arriba se han obtenido, en su orden: crudo reducido, gasóleos, A.C.P.M., queroseno, turbosina, nafta y gases ricos en butano y propano. Algunos de estos, como la turbosina, queroseno y A.C.P.M., son productos ya finales. Las demás corrientes se envían a otras torres y unidades para someterlas a nuevos procesos, al final de los cuales se obtendrá el resto de los derivados del petróleo. Así, por ejemplo, la torre de "destilación al vacío" recibe el crudo reducido de la primera etapa y saca gasóleos pesados, bases parafínicas y fondos. 


\section{“Proceso de destilación del petróleo". Ecopetrol.2013}

La Unidad de Craqueo Catalítico o Cracking recibe gasóleos y crudos reducidos de crudos livianos para producir fundamentalmente gasolina y gas propano. Las unidades de Recuperación de Vapores reciben los gases ricos de las demás plantas y sacan gas combustible, gas propano, propileno y butano.

La planta de mezclas es en últimas la que recibe las distintas corrientes de naftas para obtener la gasolina motor, extra y corriente. La unidad de Aromáticos produce a partir de la nafta: tolueno, xilenos, benceno, ciclohexano y otros petroquímicos. La de Parafinas recibe destilados parafínicos y nafténicos para sacar parafinas y bases lubricantes. De todo este proceso también se obtienen azufre y combustóleo. El combustóleo es lo último que sale del petróleo. Es algo así como el fondo del barril. El gas natural, rico en gases petroquímicos, también puede procesarse en las refinerías para obtener diversos productos de uso en la industria petroquímica.

El Complejo Industrial de Barrancabermeja cargó en el 2006 un promedio diario de 231.969 barriles para procesarlos y obtener los combustibles y demás derivados. La refinería más grande del mundo, que es el Complejo Refinador de Paraguaná en el noroccidente de Venezuela es en realidad la suma de 3 refinerías que en su conjunto procesan una dieta de crudo diario superior a los 900 mil barriles.

Colombia es considerado internacionalmente un país modesto en la industria de los hidrocarburos pero tiene una balanza comercial positiva en petróleo, gas y en general en recursos minero energéticos.

La política petrolera del gobierno colombiano se ha concentrado en los últimos años en la atracción de inversión extranjera y ampliación de las actividades de sísmica, exploración y explotación con el propósito de mantener la autosuficiencia del país y aumentar las reservas. Además el Ministerio de Minas y Energía incluye entre los objetivos de política "la competencia en los sectores de transporte, almacenamiento y distribución de combustibles líquidos derivados del petróleo, la formación de precios de los energéticos mediante la adopción de esquemas de mercados y la promoción del desarrollo y la competencia en el mercado de biocombustibles"

Colombia es un país con solo el 0,1\% de las reservas mundiales de petróleo pero adquiere alguna importancia en la región por el potencial que se reconoce a su configuración geológica, por las reservas de gas y la coyuntura geopolítica latinoamericana, particularmente en lo relacionado con Venezuela. Una característica importante del panorama de las reservas es la alta concentración en los grandes yacimientos de Arauca y Casanare que están manejados por contratos de asociación con la Occidental Petroleum Co y la BP en los cuales el Estado por medio de Ecopetrol tiene hasta un $30 \%$. Situación similar se registra en las reservas de gas que en el $97 \%$ se encuentran en dos contratos de asociación en Guajira y Casanare con la Texas y BP7 La distribución de las reservas probadas por cuenca muestra en 2004 que el $85 \%$ se ubica en los Ilanos, valle del Magdalena Medio, cordillera nororiental y Catatumbo con un peso dominante de las dos primeras. $Y$ en cuanto a gas la casi totalidad se encuentra en la Guajira y en la cordillera nororiental. La curva de reservas en esta década muestra un balance negativo entre producción y nuevos hallazgos que se explica por el peso de los macro yacimientos descubiertos en los noventa y el descenso de la actividad exploratoria entre 1994 y 2001. Sin embargo, el impulso de la inversión sísmica y en exploración en los últimos años en un escenario de precios radicalmente distinto al de los años noventa, ha permitido al gobierno proyectar la recuperación de las reservas. En esas proyecciones se incluyen nuevas regiones que se consideran potencialmente explotables, especialmente en la costa pacífica chocoana, Alto Magdalena, Putumayo y zonas del Caribe en los departamentos de Sucre, Córdoba y Magdalena, incluida la plataforma marina.

"Si el petróleo representa hoy un problema, esperemos a que pasen veinte años: será una pesadilla"

Jeremy Rifkin 